Ein Tag Notfalldienst ...

Nach dem Aufruf im Namen der Kommission «Recherche et réalisation en médecine appliquée» (RRMA) der SAMW durch Prof. Hans Stalder, Genf, erschienen in der Schweizerischen Ärztezeitung Nr. 10 vom 6. März 2002, publizieren wir hier weitere Texte. Informationen erhalten Sie unter folgender E-Mail-Adresse: hans.stalder@hcuge.ch.

Un jour de garde...

A la suite de l'invitation par le Professeur Hans Stalder, Genève, au nom de la Commission Recherche et réalisation en médecine appliquée RRMA de l'ASSM, paru dans le Bulletin des médecins suisses no 10 du 6 mars 2002, nous publions d'autres textes. Des informations peuvent être obtenues en écrivant à l'adresse e-mail suivante: hans.stalder@hcuge.ch.

\title{
Ein Tag Notfalldienst ... in den Zürcher Stadtkreisen 4, 5 und 9
}

\author{
D. Schlossberg
}

Korrespondenz:

Dr. med. Daniel Schlossberg Bachmattstrasse 53

CH-8048 Zürich
Es ist Freitag, der 11. Januar 2002. Ich erwache mit den Weckzeichen von Radio DRS 1 - die Senderwahl erfolgt einzig nach Gesichtspunkten des möglichst optimalen Empfangs und der Vermeidung unnötiger Aufregungen. Wie immer ist mein Puls an solchen Tagen - und dies in Ruhe - kaum zählbar hoch, 130 Bits pro Minute mögen es sein, und ich denke, wenn doch nur mein Internetanschluss so effizient feuerte. Immerhin, auch das ist Ritual, liegt der Betablocker - aus Kostengründen ateno-basan - bereit, und ich mache die für mich optimale Frequenzkorrekturdosis von $125 \mathrm{mg}$ bioverfügbar. Wiederum hat Herr Schönenberger nur gerade die 25-mgDosierung als pro medico hinterlassen und so schlucke ich aus Kostengründen 5 Tabletten. Das heisst für mich, um die Pillen nicht sofort zu regurgitieren, fünf Gläser Wasser nachtrinken. Nach etwa 75 Minuten sinkt mein Puls in für mich messbare Bereiche ab und pendelt sich bei 84 pro Minute ein. Ich bereite einen Espresso zu im Wissen um den beruhigenden Effekt, den das Koffein auf mein Nervenkostüm ausübt.

Während das Wasser durch den Kolben durchgepresst wird, klingelt ein erstes Mal das Telefon und es meldet sich Schwester H. des Ärztefons, auch etwas gestresst und deshalb ohne Entschuldigung, schon vor Beginn der Sprechstunde stören zu müssen. Es geht um eine 46jährige Dame, welche Krämpfe im ganzen Oberkörper beklagt und eine - selbstredend frustrane - Selbstbehandlung mit kalten und wechselweise warmen Wickeln, die sie auf die Stirn plaziert, durchgeführt hat, ehe der Familienrat, nachdem das Geschehen vor 2 Tagen bereits begonnen hatte, sich der Nummer des Hausarztes erinnert hatte. Da zu dieser Zeit die Praxisräume üblicherweise verwaist sind, kam eine Bandmitteilung des Hausarztes, der empfahl, direkt die Telefonnummer des Ärztefons anzuwählen, sei es, wie es sei.

Ich machte mich auf den Weg, noch mit der zusätzlichen Information gefüttert, dass es sich um eine adipöse Diabetika handle. Ich machte einige differentialdiagnostische Überlegungen und obwohl die Patientin nicht weit von meinem Wohnort entfernt wohnte, blieb noch genügend Zeit, irgendwelche Sportresultate mitzuhören. In der Wohnung der Patientin eingetroffen, wurde mir sofort klar: nicht nur der Name der Frau tönt italienisch, auch die Sippenverhältnisse sind es - ich komme darauf zurück. Wie sich nun herausstellt, rief die Tochter nach dem Notfallarzt, weil es seit einer Stunde zu einer Schmerzexazerbation gekommen ist, und das tat den Angehörigen fast mehr weh als der Patientin. Immerhin: sie hatte Krämpfe im ganzen Bauch, musste erbrechen und teilte mit Tausenden von anderen Frauen das Leiden des zyklischen Wendepunkts. Weitere zum digestiven Trakt erhältliche Informationen waren, dass zweimal Durchfall eingetreten sei und eine ähnliche Episode schon vor 3 oder 4 Tagen stattgefunden habe.

Als ich mit der Anamnese eben fertig geworden war und zur Untersuchung übergehen wollte, wurde mir ganz anders, schwarz vor den Augen und ich kollabierte beinahe. Mein Ruhe- 
puls betrug noch 30 pro Minute und ich war total benebelt. Der Ehemann rief über 144 die Sanität, Blaulicht war gefordert, und 10 Minuten später der Puls durch Atropingabe auf 45 angestiegen. Im Spital eingetroffen beugten sich viele bekannte und unbekannte Gesichter über mich und Ärzte tuschelten miteinander; schliesslich erklärte der Chefarzt, den ich naturgemäss gut kenne, ich hätte einen totalen AV-Block und erhielte nun einen provisorischen Schrittmacher. Ob ich denn nie ein Ruhe-EKG vor jeweiliger Einnahme der satten Betablockerdosierung von $125 \mathrm{mg}$ Atenolol geschrieben hätte ...? Dort hätte ich doch sehen müssen, dass eine vorbestehende Überleitungsstörung vorliege. Beschämt gab ich kleinlaut $\mathrm{zu}$, mich für herzgesund gehalten $\mathrm{zu}$ haben und wollte in den Boden versinken.

Glücklicherweise erwachte ich in diesem Augenblick und fühlte meinen Puls, der 74 pro Minute wie meistens in Ruhe betrug. Ich erinnerte mich der Patientin, die ich vorgestern nachts gemeinsam mit meiner Frau besucht hatte. Und dass ateno-basan nur in 50- und 100-mg-Dosierung auf dem Markt ist, was mir definitiv Gewissheit gab, nun wach zu sein. Nach der Anamnese folgte die Untersuchung und diese war wie so oft bei Adipösen mit einem Bauchproblem eine Nagelprobe. Nach kurzem internistischem Kränzli entschlossen wir uns zur Spitaleinweisung mit folgender Beurteilung: 1. V.a. symptomatische Cholezystolithiasis, eventuell Cholezystitis. 2. Diabetes mellitus Typ 2 mit Adipositas und 3. Arterielle Hypertonie. Die Standardbehandlung durch den Hausarzt bestand in Daonil 2-0-1, Glucophage forte 1-0-1 und Tenormin $100 \mathrm{mg}$ 1-0-0. Um das Bild nicht zu verfälschen, applizierten wir keine Analgetika.

Es ist nun definitiv Freitag, der 11. Januar 2002, und der Dienst beginnt erst 12 Uhr mittags. Inzwischen steigt mein Puls frühmorgens selbst an Diensttagen kaum an und den Espresso kann ich mir in Ruhe zubereiten und zuführen. Unser Notfalldienst in der Stadt Zürich ist durch eine hohe Ärztedichte abgefedert und an einem Freitag arbeiten die meisten Kolleginnen und Kollegen. So erscheint der erste Notfall erst nachmittags um etwa 3 Uhr. Das Nasenbein ist gebrochen, und es geschah gestern beim Fussballspiel. Da ich als Internist nicht gipse, überweise ich den Patienten auf die Notfallstation des nahegelegenen Triemlispitals zu Händen des Kollegen der ORL. Ich verspreche mir davon, dass mein Eindruck, das Nasenseptum sei etwas in Schieflage geraten, korrigiert oder allenfalls und andernfalls das Nasenseptum aufgerichtet werden kann. Ich kann es vorwegnehmen: Ich erhalte nie einen Bericht, und so habe ich nichts dazugelernt. Übrigens nebenbei: Der Datenschutz gebietet, dass nur der Hausarzt, nicht aber der Einweisende orientiert werden darf. Eine sinnige Regelung ganz im Sinne der Qualitätssicherung!

Die Sprechstunde neigt sich dem Ende zu, pünktlich um 17 Uhr löst die Raumpflegerin die medizinische Praxisassistentin - welch scheussliche Berufsbezeichnung! - ab. Nur ich wirke weiter und mache mich daran, Pendenzen abzutragen. Dies sollte sich als folgenschwerer Fehler erweisen. Aber um 17 Uhr sind die Strassen eh meistens verstopft.

Kaum zu Hause eingetroffen, werde ich zu einem unklaren Abdomen gerufen. Da es Abend und meine Frau vom Fach ist - sie praktiziert mit mir zusammen in unserer Gemeinschaftspraxis -, begleitet sie mich zu diesem 78jährigen Herrn, der an der Alzheimer Krankheit und nun an Bauchschmerzen leidet. Von der Ehefrau erfahren wir, dass Herr K. mindestens dreimal erbrochen hat, und er selbst gibt bandförmige Schmerzen auf Höhe Bauchnabel an. Trotz all unserer Bemühungen hören wir keine Darmgeräusche und zusammen mit der Anamnese denken wir trotz weichem Bauch an ein gedecktes perforiertes Ulcus, an eine mesenteriale Ischämie und an eine Pankreatitis und weisen diesen Herrn mit der Sanität und mit der Beurteilung eines paralytischen Ileus ins Spital ein. Weil es uns interessiert, werde ich mich zwei Tage später erkundigen, was die weiteren Abklärungen ergeben haben. Wir fahren nach Hause, einem «loup de mer», oder war es nicht viel eher ein St. Pierre, entgegen.

Unter der Haustür geht das Telefon, der nächste Einsatz ruft. Eine 87jährige Dame hat Fieber und katheterisiert sich seit Jahren wegen Restharns selber. Wir sehen uns erneut, dieses Mal bei Verdacht auf eine aszendierende Pyelonephritis durch unsachgemässe Selbstkatheterisierung, zur Hospitalisierung gezwungen. Wie meist bei solchen Hausbesuchen vergeht eine Stunde, bis wir wieder zu Hause angelangt sind.

Die Zubereitung des Fischgerichts nimmt meine Frau nun in Angriff, während ich mich mit dem Gedanken anfreunde, das könnte es für heute gewesen sein. Nach knappen 20 Minuten ruft uns der Sohn eines 64jährigen Herrn zu Hilfe, der zunehmende Atemprobleme habe. Mehrere Ärzte hätten ihn in den letzten Tagen gesehen und heute weilte man auf der Notfallstation des Universitätsspitals, wo man darauf hingewiesen worden sei, mehrere Stunden Wartezeit in Kauf nehmen zu müssen. So kehrte der iranische Patient ungesehen nach Hause zurück, wo er nun nicht mehr die Bettstatt verlassen 
könne. Der Sohn denkt, dass ein lebensbedrohlicher Zustand vorliege. Ich frage nach Alter und Beruf des Anrufers zur Abschätzung der Glaubwürdigkeit der gemachten Angaben und erfahre, dass er 27jährig und Buchhalter bei einer Bank sei. Von uns zum Patienten sind es unter Einhaltung von Tempo 50 bzw. 30 in den Wohnzonen zu dieser späten Abendstunde ohne Werkverkehr etwa 15 bis 20 Minuten. Ich denke, dass eine Hospitalisation unumgänglich sein wird, da Herr R. ohne Fremdhilfe nicht mehr aufstehen könne, denke an verschiedenes, zum Beispiel eine Addisonkrise und weise direkt ein unter Avisierung der Sanität. Von dieser erhalte ich den Rückruf, das sei kein Fall für ein Akutspital, die Vitalparameter seien alle gut, und sie dächten an ein psychogenes Problem. Der Patient sei sitzund, wenn er wolle, auch gehfähig. Ich habe gründlich falsch gedacht und hätte stutzig werden sollen ob der vorausgegangenen Ärzteodyssee. Dieser Fall ärgert mich und vergällt mir das Nachtessen - hier trifft der Ausdruck zu: es ist Nacht - gründlich! So weiss ich eben nicht mehr, wie der Fisch hiess ...

Das sollte gleich völlig belanglos werden, denn eine ältere Dame rief um Hilfe, bei ihr sässe ein Herr als Gast zu Tische, der seit einer Stunde nicht mehr spreche und nun auch je länger, je weniger schnaufe. Wir verlieren keine Zeit und eilen hin, der Tatort ist nahe gelegen, nach 5 Minuten sind wir eingetroffen. Die Küchenszene hätte gut in ein Werk von Beckett oder Kafka gepasst: Ein Herr mit fahlem Kolorit sitzt nicht ansprechbar am Tisch, mit dem Oberkörper leicht nach vorne gegen die Tischkante geneigt, über einem Teller mit inzwischen kaltem Spiegelei und Bratkartoffeln. Laut der Gastgeberin, die diesem Treiben seit gut einer Stunde tatenlos zugesehen hat, ehe sie zum Telefonhörer griff und Hilfe anforderte, habe der Gast vom 5. Stockwerk schon vor Eintreffen bei ihr - und das ist in der 7. Etage - reichlich Weisswein konsumiert, was er sich nicht so gewohnt sei. Es pressiert. Kein Karotispuls, nur noch selten ein Atemzug. Ich bin froh, dass wir zu zweit sind, Herr M. muss runter vom Stuhl auf den Boden, was uns Schweissperlen auf die Stirne treibt. Die Platzverhältnisse sind eng, der Mann ist schwer und er atmet schwer. Immerhin scheint in horizontaler Position doch ein Kreislauf existent und die Atemfrequenz nimmt zu. Die üblichen Massnahmen folgen: Venöser Zugang, 5\% Glukoseinfusion, Anforderung der Sanität, die rasch im Haus, aber lange nicht in der richtigen Wohnung eintrifft, weil die Informationsübermittlung, dass der Patient zu Besuch bei Frau R. 2 Stockwerke höher weilt, Schaden leidet. Was wir dann erleben, hat uns verblüfft: Herr M. beginnt sich zu wehren, will sich die Leitung ziehen und ist schliesslich so wach, dass er Undeutliches spricht und sich der Hospitalisation widersetzt. Es gelingt noch, ihn im Notfall abzuliefern, dort kann ein Blutalkoholspiegel von 2,7\%o ermittelt werden, doch alsbald entwischt er dem geschäftigen Treiben auf der Notfallstation.

Wir fahren nach Hause und ich sehe mich genötigt, dem Sohn jenes Herrn mit dem psychogenen Ausnahmezustand zurückzurufen. Der Bruder nimmt meinen Anruf entgegen und berichtet, dass es zwischenzeitlich dem Vater besser gehe. Die Vorwürfe, ich sei zu bequem gewesen, den Hausbesuch zu machen, weise ich entschieden zurück. Vielleicht war es auch einfach eine Fügung, denn die vita minima mit Alkohol quantum satis an Bord wurde wieder zur vita maxima. Und das ist vielleicht doch der Augenblick, ein Wort zu Ethnien zu verlieren: Menschen italienischer Provenienz haben oft laute Schmerzen, aber wenn die Sippe - und die gibt es sympathischerweise im wahren Sinne des Wortes bei unseren südlichen Nachbarn noch um Hilfe ruft, so hat das meistens Hand und Fuss. Etwas anders verhält es sich mit Immigranten aus dem Nahen und Mittleren Osten. Die Elterngeneration ist oft schlecht assimiliert und Normen und Werte sind nicht identisch mit den unseren. Das muss bei der Beurteilung immer berücksichtigt werden - und das tat ich in jenem Fall leider ungenügend.

Inzwischen ist Mitternacht vorbei und es ist Zeit, schlafen zu gehen. Wir profitieren davon, dass zwischen 24 und 6 Uhr ein Nachtarzt für die ganze Stadt im Einsatz steht, und wir nur Pikett leisten. In all den Jahren ist es nie vorgekommen, dass wir den Nachtarzt hätten entlasten müssen.

Am Samstagvormittag ist es für einmal ausgesprochen ruhig, erst gegen $11 \mathrm{Uhr}$ werde ich zu einem 1914 geborenen Herrn gerufen, der Bauchschmerzen seit drei Tagen rund um den Nabel hat. Duplizität der Fälle. Auch in diesem Fall gestaltet sich die Anamnese zeitraubend, doch schliesslich denke ich, dass es doch mehr als eine banale Gastroenteritis mit etwas Durchfall und vor allem schlafraubenden Bauchschmerzen sein könnte, und weise den Patienten der nahe gelegenen chirurgischen Notfallstation zu. Dort erhält Herr K. einen hohen Einlauf, kehrt nach Hause zurück, um 2 Tage später für 14 Tage stationär aufgenommen zu werden, da es nach dem hohen Einlauf nicht besser ging, und die Mittelbauchschmerzen, die mich an eine Bride bei Zustand nach diversen abdominalen Eingriffen initial hatten denken lassen, weitere Untersuchungen nötig machten. Schliesslich 
blieb es bei Abdominalschmerzen im Rahmen einer Koprostase, nebenbefundlich wurden noch eine nicht stenosierende Sigmadivertikulose und drei kleine Polypen im Zökum festgestellt. Eine Koprostase, die teuer zu stehen kam und mit der Empfehlung einer ausreichenden Stuhlregulation inskünftig zu vermeiden gesucht wird. Ja, und was häufig ist, ist häufig: der AlzheimerPatient mit Bauchschmerzen litt an Koprostase. Duplizität der Fälle, wie gesagt.

So blieb noch ein Abdomen, das der 46jährigen Italienerin, welche wir eingewiesen hatten. Es handelte sich um eine Einblutung in die Leber bei hochgradigem Verdacht auf ein Leberadenom, differentialdiagnostisch hepatozelluläres Karzinom. Die Hemihepatektomie wurde elektiv geplant, die definitive Histologie wird Klärung bringen.

Eigentlich habe ich den Austrittsbericht nach Grundsätzen des angewandten Personendatenschutzes nicht zugute, doch bin ich allemal froh, wenn es damit nicht alle so ganz genau nehmen. Denn für uns Notfallärzte ist der Feedback, was unsere Beurteilungen - meist beim Patienten zu Hause und nur mit den Hilfsmitteln der Anam- nese und klinischen Untersuchung erhoben betrifft, die einzige Qualitätskontrolle unseres Tuns. Die aszendierende Pyelonephritis war nachweislich eine solche bei Retentionsblase und Selbstkatheterisierung. Dass dies der erste Infekt nach neun Jahren der täglichen Selbstkatheterisierung war, hat unseres Erachtens weniger mit den sterilen Kautelen, wie bei dieser Massnahme eigentlich vorzusehen, als mit dem Umstand zu tun, dass der Mensch von Natur aus zäh ist. Wir hätten uns vorgestellt, dass inskünftig eine Gemeindeschwester für eine sachgemässe Handhabung sorgt. Im Fall des zu erwartenden nächsten invasiven Harnwegsinfekt gibt es wieder einen Notfallarzt, der gerufen werden kann!

Ein Nachwort: Vor wenigen Tagen erhielt ich eine Karte jenes Mannes, der infolge toxischer Einwirkung von weissem Rebensaft nicht mehr ganz munter gewirkt hatte. Er teilte mit, nachdem er einen Kontoauszug zugestellt erhalten hatte, wann er die Rechnung zu bezahlen gedachte und vermerkte kurz und geläutert: «Herzlichen Dank». 